\title{
Construcción del modelo científico escolar de arribo como eje directriz para el diseño de una secuencia didáctica sobre el cambio químico
}

Miguel, Isabel' , Merino, Cristian², Reyes, Flor ${ }^{3}$ y López-Valentín, Dulce ${ }^{4}$

Categoría 2. Trabajo de investigación (en proceso)

\section{Resumen}

Se propone la construcción de un 'Modelo Científico Escolar de Arribo' (Mcea), que funcione como hipótesis directriz al proporcionar criterios teóricos y metodológicos para diseñar una secuencia didáctica que posibilite la comprensión del cambio químico en el nivel secundaria. Se describe la forma de proceder para su elaboración, las ventajas que este modelo ofrece y su aplicación en la reacción de combustión como ejemplo de reacción química.

Consideramos que la modelización es una alternativa viable que facilita a los estudiantes de secundaria la comprensión del cambio químico, para explicar los fenómenos químicos cotidianos de su entorno y en consecuencia, actuar e intervenir en ellos aplicando el conocimiento desarrollado mediante la actividad científica escolar.

\section{Palabras clave}

Modelización, cambio químico, combustión, enseñanza de la química, ciencia escolar.

\section{Objetivo}

Construir el Modelo Científico Escolar de Arribo (MCEA) que proporcione los criterios teóricos y metodológicos para el diseño y desarrollo de una Secuencia Didáctica basada en la modelización, cuya intención será que los estudiantes de tercer grado de secundaria comprendan el cambio químico mediante el fenómeno de la combustión.

\footnotetext{
${ }^{1}$ Universidad Pedagógica Nacional-Ajusco (México); Isabel_miguel_lopez@hotmail.com

2 Pontificia Universidad Católica de Valparaíso (Chile); cristian.merino@ucv.cl

${ }^{3}$ Universidad Nacional Autónoma de México; freyes@g.upn.mx

${ }^{4}$ Universidad Pedagógica Nacional-Ajusco (México); dvalentín@upn.mx
} 
Revista Tecné, Episteme y Didaxis: TED. Año 2014, Número Extraordinario. ISSN Impreso: 0121-3814, ISSN web: 2323-0126

Memorias, Sexto Congreso Internacional sobre Formación de Profesores de Ciencias. 08 al 10 de octubre de 2014, Bogotá

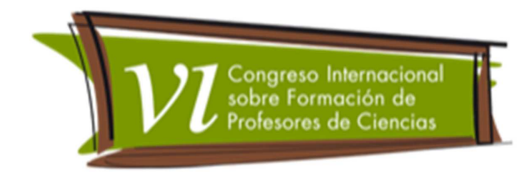

\section{Marco teórico}

Vivimos rodeados de cambios químicos que ocurren en todo momento y en todo lugar, conforme la humanidad logró el conocimiento de las fórmulas y leyes que rigen las combinaciones químicas ha querido entender la naturaleza de las mismas para poder sujetarlas a su voluntad. Sin embargo, el cambio químico siempre ha resultado dificil de comprender y ha significado un reto para los docentes lograr que los estudiantes alcancen aprendizajes significativos (Izquierdo et al., 2007). A continuación se enlistan algunos de los factores que dificultan la comprensión del cambio químico.

1. La abstracción de los contenidos (Johnstone, 1982; Izquierdo, 2004; Izquierdo et al., 2007).

2. La persistencia de las ideas previas de los alumnos aún después de la instrucción escolarizada (Driver et al., 1988).

3. El uso de un lenguaje de símbolos y fórmulas (Izquierdo, 2004; Izquierdo et al., 2007).

4. La visión macroscópica que tienen los estudiantes sobre el cambio químico considerando a la materia de naturaleza continua (Hatzinikita et al., 2005; Merino e Izquierdo, 2011).

Estos factores, permiten comprender que existen obstáculos epistemológicos difíciles de superar. Con este trabajo, por lo tanto se pretende contestar concretamente la siguiente pregunta de investigación: ¿De qué forma puede intervenir el docente para que sus estudiantes comprendan el cambio químico, se acerquen a las explicaciones científicas y puedan aplicar los aprendizajes en su vida cotidiana?

Desde un enfoque constructivista sobre el aprendizaje y la enseñanza de la química, se considera que la comprensión de los fenómenos químicos se posibilita a través de la construcción de modelos; entendidos éstos como aquellas representaciones dinámicas con carácter explicativo y predictivo en la que participan determinadas entidades, relaciones y condiciones (Schwartz, 2009). 
Revista Tecné, Episteme y Didaxis: TED. Año 2014, Número Extraordinario. ISSN Impreso: 0121-3814, ISSN web: 2323-0126 Memorias, Sexto Congreso Internacional sobre Formación de Profesores de Ciencias. 08 al 10 de octubre de 2014, Bogotá

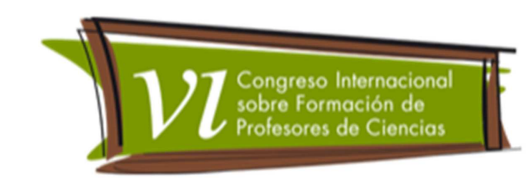

Schwartz (2009) y Merino e Izquierdo (2011) argumentan que los modelos pueden representarse de manera simple -destacando sólo lo esencial que sirva para explicar el fenómeno de interés- mediante maquetas, dibujos, fórmulas, símbolos, diagramas o un hecho ejemplar lo que facilita la comprensión de los fenómenos del entorno y permite hacer predicciones, diseñar experimentos así como tomar decisiones informadas sobre qué hacer en una determinada situación del mundo real.

Se plantea por lo tanto, que la modelización es útil para comprender el cambio químico, y que por su carácter semántico un modelo puede servir de referencia para explicar fenómenos similares (Giere, 1999a). A través de la elaboración de modelos se movilizan saberes que permiten pensar, describir, predecir, actuar (Izquierdo, 2007) y los estudiantes pueden experimentar, representar y comunicar aspectos sobre entidades teóricas no visibles o abstractas, que constituyen el lenguaje simbólico de la química.

Giere (1999a), sugiere emplear fenómenos que se correspondan con la realidad, de esta forma será útil que los estudiantes puedan explicarse los fenómenos del entorno y aplicar sus aprendizajes durante toda la vida. Por ello se ha decidido trabajar con el fenómeno de la combustión -como ejemplo-, por representar un fenómeno cotidiano para los estudiantes, ser relativamente fácil de reproducir en condiciones de laboratorio escolar y porque la energía que en esta reacción se genera es ampliamente utilizada por el hombre en forma de energía calorífica y luminosa o trabajo, para satisfacer necesidades tales como la cocción de los alimentos, la calefacción, el transporte o la puesta en marcha de diversos procesos industriales.

Al considerar entonces que el fenómeno de la combustión es un buen ejemplo para comprender el cambio químico, se pretende diseñar una secuencia didáctica en donde se utilice el Mcea, como instrumento de diseño.

De acuerdo con López-Mota y Rodríguez (2013) para diseñar una Secuencia Didáctica con base en el MCEA, es necesario tomar en cuenta el modelo de partida de los alumnos -al cual en esta propuesta hemos llamado 'Modelo Explicativo Inicial' (Mei)-. Así mismo habrá que construir otros tres modelos más: un 'Modelo Curricular' (Mcu), un Modelo Científico (Mci) y el Mcea.

Para poder operativizar los tres modelos anteriores, será necesario "formular los enunciados de los distintos modelos en una forma homogénea, de tal manera que sean comparables" (López-Mota y Rodríguez, 2013:201 1). La forma en que los 
Revista Tecné, Episteme y Didaxis: TED. Año 2014, Número Extraordinario. ISSN Impreso: 0121-3814, ISSN web: 2323-0126 Memorias, Sexto Congreso Internacional sobre Formación de Profesores de Ciencias. 08 al 10 de octubre de 2014, Bogotá

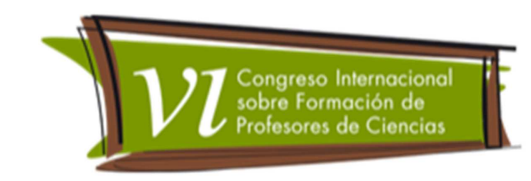

modelos se hacen homogéneos, es expresarlos en términos de elementos, relaciones y normas de operación (Schwartz, 2009). Para este estudio las normas de operación serán entendidas como condiciones y los elementos del modelo serán llamados entidades, para evitar confusión con los elementos químicos. A continuación se definen los citados modelos:

\section{Modelo Explicativo Inicial}

En esta propuesta se entenderá como Mei a la forma en que los estudiantes se explican el fenómeno en cuestión. El Mei, es inferido a partir de la revisión de las ideas previas -reportadas en la literatura- que el estudiantado tiene sobre dicho fenómeno. Por lo tanto, este modelo es producto de la investigación teórica.

\section{Modelo Curricular}

El Mcu se infiere a partir del planteamiento curricular de los planes y programas de estudio sobre el contenido a impartir (López-Mota y Rodríguez, 2013:2009).

\section{Modelo Científico}

El Mci se plantea una vez realizada la revisión de libros de nivel superior sobre el tema en cuestión, es el referente científico en donde se plantean las teorías, leyes y principios que dan cuenta del fenómeno en estudio.

\section{Modelo Científico Escolar de Arribo}

Este modelo es producto de la tensión entre el Mcu y el Mci, así como de la debida transposición didáctica creada para el tópico de estudio en particular.

El Mcea es una propuesta de carácter teórico-metodológica y se postula como una hipótesis directriz que proporciona los criterios orientadores necesarios para diseñar una Secuencia Didáctica. Es un constructo que funciona también como medio para evaluar los avances de los estudiantes en la comprensión del fenómeno de estudio, logrados al aplicar la secuencia diseñada. Esta evaluación es posible a través del 'Modelo Alcanzado' (López-Mota y Rodríguez, 2013).

De acuerdo con Miguel (2014) se considera que en el contexto mexicano, el avance en el aprendizaje partiendo del Mei hacia el Mcea es similar a lo que García y Sanmartí (2006) han trabajado como evolución en el aprendizaje, cuando avanzan los estudiantes de un modelo simple y concreto a un modelo complejo y abstracto. De igual forma, es similar a la idea que tanto Salinas et al. 
Revista Tecné, Episteme y Didaxis: TED. Año 2014, Número Extraordinario. ISSN Impreso: 0121-3814, ISSN web: 2323-0126

Memorias, Sexto Congreso Internacional sobre Formación de Profesores de Ciencias. 08 al 10 de octubre de 2014, Bogotá

(2013) como Talanquer (2013) han presentado bajo la forma de modelo Ancla Inicial y Ancla Final para planear Progresiones de Aprendizaje. (ver figura 1).

Figura 1. Similitud del MCEA con el Modelo Complejo-Abstracto propuesto por García y Sanmartí (2006) y con el Modelo de Ancla Superior propuesto por Salinas (2013) y Talanquer (2013). (Fuente:

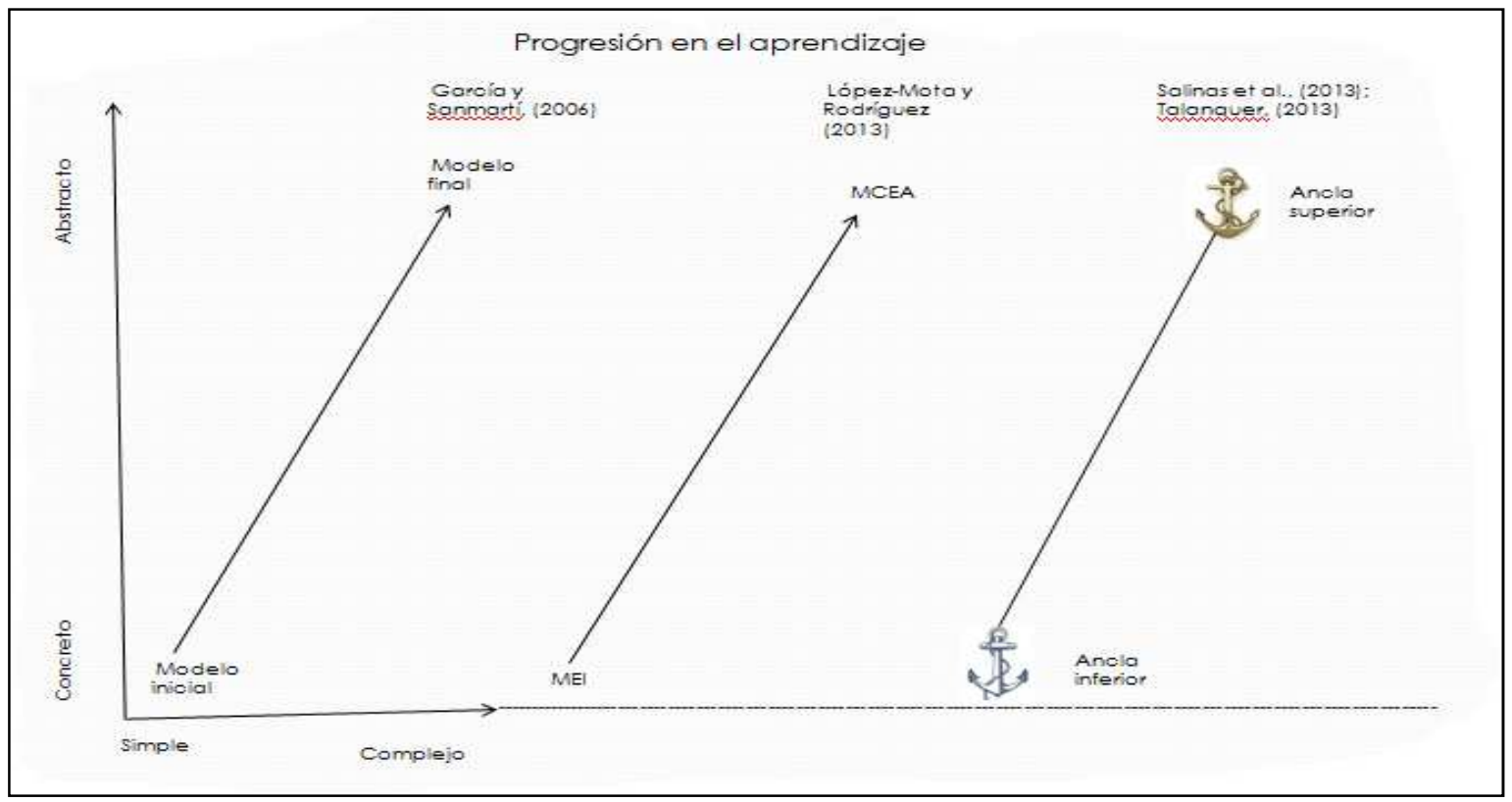

Miguel, 2014).

\section{Metodología}

1. Indagar en la literatura las ideas previas que sobre el cambio químico, (combustión), tienen los estudiantes de 14 a 15 años, e inferir el Mei.

2. Revisar el tema de la combustión en los planes y programas oficiales y en los libros de texto de la asignatura Ciencias III (con énfasis en química). A partir de tal revisión elaborar el Mcu.

3. Revisar en libros de nivel superior el tema de cambio químico y la combustión, con ello plantear el Mci.

4. Expresar todos los modelos en términos de entidades relaciones y condiciones para que sean homogéneos. 
Revista Tecné, Episteme y Didaxis: TED. Año 2014, Número Extraordinario. ISSN Impreso: 0121-3814, ISSN web: 2323-0126

Memorias, Sexto Congreso Internacional sobre Formación de Profesores de Ciencias. 08 al 10 de octubre de 2014, Bogotá

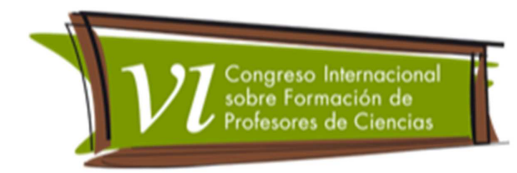

5. Tensionar el Mcu con el Mci y seleccionar mediante la transposición didáctica las entidades, relaciones y condiciones necesarios para la construcción del Mcea.

\section{Resultados}

En la literatura se encuentran gran cantidad de ideas previas que los alumnos tienen sobre el cambio químico y la combustión. Para este estudio sólo se reportan cinco ejemplos considerados como representativos.

1. "Cuando algo se quema, queda la ceniza y algo desaparece y creo que pesa menos" (Driver et al., 1988).

2. "Madera, carbón, papel, aire, se transforman en otra sustancia o en nada sin conservación de la sustancia" (Méheut et al., 1985).

3. "Una cinta de hierro es transmutada a carbón por la combustión" (Anderson, 1990).

4. "Los materiales cambian de forma o de organización pero siguen siendo la misma sustancia" (Hatzinikita et al., 2005).

5. "Los alumnos consideran que la materia es continua y sus respuestas están relacionadas con la transmutación" (Merino e lzquierdo, 2011).

La idea de fondo que se aprecia es que en la combustión los materiales desaparecen, se modifican $\mathrm{o}$ se transmutan, la materia es continua y la explicación del cambio químico se da en términos macroscópicos. A partir de esta idea, se infiere el Mei.

En la tabla 1 se muestran las entidades, relaciones y condiciones tanto del Mei como del Mcu y del Mci para el fenómeno de la combustión.

La tabla 2 muestra el Mcea, obtenido al tensionar el Mcu y el Mci.

Tabla 1. Entidades relaciones y condiciones del MEl, MCU Y MCl.

\begin{tabular}{|l|l|l|l|}
\hline & Mei & Mci & Mcu \\
\hline Entidades & Material o sustancia & Reactivos: & Sustancias \\
\hline
\end{tabular}


Revista Tecné, Episteme y Didaxis: TED. Año 2014, Número Extraordinario. ISSN Impreso: 0121-3814, ISSN web: 2323-0126

Memorias, Sexto Congreso Internacional sobre Formación de Profesores de Ciencias. 08 al 10 de octubre de 2014, Bogotá

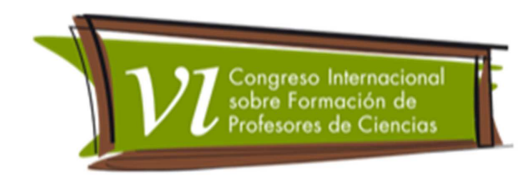

\begin{tabular}{|c|c|c|c|}
\hline & $\begin{array}{l}\text { que se quema } \\
\text { Flama } \\
\text { Humo } \\
\text { Cenizas }\end{array}$ & $\begin{array}{l}\text { combustible y comburente } \\
\text { Oxidante, Reductor } \\
\text { Productos: } \\
\text { Dióxido de carbono, monóxido } \\
\text { de carbono, agua, otros gases } \\
\text { (depende del combustible) }\end{array}$ & $\begin{array}{l}\text { a)Reactivos: combustible, } \\
\text { comburente (oxígeno) } \\
\text { b) Productos: dióxido de } \\
\text { carbono y agua. }\end{array}$ \\
\hline Relaciones & $\begin{array}{l}\text { Transmutación } \\
\text { Desaparición } \\
\text { Modificación }\end{array}$ & $\begin{array}{l}\text { Combinación química (entre } \\
\text { reactivos) } \\
\text { Transformación (de reactivos a } \\
\text { productos) } \\
\text { Liberación (de energía) }\end{array}$ & $\begin{array}{l}\text { Transformación (de } \\
\text { reactivos a productos) }\end{array}$ \\
\hline Condiciones & No hay condiciones & $\begin{array}{l}\text { Energía de activación para } \\
\text { iniciar el proceso. } \\
\text { Comburente: oxígeno o una } \\
\text { fuente del mismo } \\
\text { La masa y la energía se } \\
\text { conservan }\end{array}$ & $\begin{array}{l}\text { El comburente es el } \\
\text { oxígeno } \\
\text { Conservación de la masa }\end{array}$ \\
\hline
\end{tabular}

Tabla 2. Entidades, relaciones y condiciones del Mcea.

\begin{tabular}{|c|c|c|}
\hline Entidades & Relaciones & Condiciones \\
\hline $\begin{array}{l}\text { a) Reactivos: } \\
\text { Materiales o sustancias que se } \\
\text { queman } \\
\text { Combustible } \\
\text { Comburente (oxígeno o una } \\
\text { fuente del mismo) }\end{array}$ & $\begin{array}{l}\text { Combinación química (entre } \\
\text { reactivos) } \\
\text { Transformación (de reactivos a } \\
\text { productos) }\end{array}$ & $\begin{array}{l}\text { Se requiere energía de activación } \\
\text { para iniciar el proceso } \\
\text { Conservación de la masa y la } \\
\text { energía }\end{array}$ \\
\hline
\end{tabular}


Revista Tecné, Episteme y Didaxis: TED. Año 2014, Número Extraordinario. ISSN Impreso: 0121-3814, ISSN web: 2323-0126

Memorias, Sexto Congreso Internacional sobre Formación de Profesores de Ciencias. 08 al 10 de octubre de 2014, Bogotá

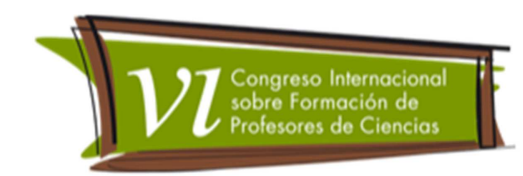

b)Productos:

Dióxido de carbono y agua. Se libera: energía luminosa y calorífica.

\section{Conclusiones}

Se ha presentado el modelo de partida de los estudiantes de 14 a 15 años sobre el cambio químico, en particular sobre la combustión.

Así mismo, se ha construido el Mcea para el fenómeno de la combustión a partir de la tensión entre el Modelo Curricular y el Modelo Científico. El Mcea es la hipótesis directriz que se tomará como base en el diseño de una secuencia didáctica para modelizar el cambio químico. La secuencia didáctica se encuentra en desarrollo.

\section{Referencias bibliográficas}

Anderson, B. (1990). Pupils' conceptions of matter and its transformations (age 1216). Studies in Science Education, 18(1), pág. 53-85.

Driver, R., Guesne, E y Tiberghien, A. (1988). Ideas cientificas en la infancia y la adolescencia. Madrid: Morata.

García, P. y Sanmartí, N. (2006). La modelización: una propuesta para repensar la ciencia que enseñamos. En Quintanilla, M. \& Adúriz-Bravo, A. (eds.). Enseñar ciencias en el nuevo milenio: Retos y propuestas. Santiago de Chile: Pontificia Universidad Católica de Chile (pág. 279-297).

Giere, R. (1999a). Del realismo constructivo al realismo perspectivo. En Enseñanza de las Ciencias, Número extra. pp. 9-13.

Hatzinikita, V., Koulaidis, V. y Hatzinikitas, A. (2005). Modeling Pupils' Understanding and Explanations Concerning Changes in Matter. Research in Science Education. 35: pág. 471-495.

Izquierdo, M. (2004). Un nuevo enfoque de la enseñanza de la química:contextualizar y modelizar. The Journal of the Argentine Chemical Society 92, (4/6), pág. 115-136. 
Revista Tecné, Episteme y Didaxis: TED. Año 2014, Número Extraordinario. ISSN Impreso: 0121-3814, ISSN web: 2323-0126

Memorias, Sexto Congreso Internacional sobre Formación de Profesores de Ciencias. 08 al 10 de octubre de 2014, Bogotá

Izquierdo, M., Sanmartí, N. y Estaña, J. (2007). Actividad química escolar: modelización metacognitiva del cambio químico. En M. Izquierdo, A. Caamaño y M. Quintanilla (eds.) Investigar en la enseñanza de la química. Nuevos horizontes: contextualizar y modelizar. Universidad Autónoma de Barcelona: España pág. 141-163.

Johnstone, A.H. (1982). Macro- and micro- chemistry. School Science Review, pág. 64 (227), 377-379.

López-Mota, A. y Rodríguez Pineda, D. P. (2013). Anclaje de los Modelos y la Modelización Científica en Estrategias Didácticas. Enseñanza de las Ciencias (Número extra), 2008 -2013.

Méheut, M., Saltiel, E. y Tiberghien, A. (1985). Pupils' (11-12 years old) conceptions of combustion. European Journal of Science Education, pág. 7, 83-93.

Merino, C. e Izquierdo, M. (201 1). Aportes a la modelización del cambio químico. En Educación Química, 22 (3), pág. 212-223.

Miguel, I. (2014). La combustión: Secuencia didáctica para modelizar el cambio químico en secundaria. Tesis de Maestría. México: Universidad Pedagógica Nacional. (En desarrollo).

Salinas, I., Covitt, B. y Gunckel, K. (2013). Sustancias en el agua: progresiones de aprendizaje para diseñar intervenciones curriculares. En Educación química, 24 (4), pág. 391-398.

Schwarz, C., Reiser, B., Davis, E., Kenyon, L., Acher, A., Fortus, D., Shwartz, y., Hug, B. \& Krajcik, J. (2009). Developing a Learning Progression for Scientific Modeling: Making Scientific Modeling Accesible and Meaningful for Learners. Journal of Research in Science Teaching, 46 (6), pág. 632-654.

Talanquer, V. (2013). Progresiones de aprendizaje: promesa potencial. En Educación Química, 24 (4), pág. 362-364. 